\title{
Special issue on multi-modal information learning and analytics for smart city
}

\author{
Zheng $X u^{1} \cdot$ Qingyuan Zhou ${ }^{2}$
}

Published online: 25 February 2020

c) Springer-Verlag GmbH Germany, part of Springer Nature 2020

We are living in the era of data deluge. Meanwhile, the world of big data exhibits a rich and complex set of cross-media contents, such as text, image, video, audio and graphics. Thus far, great research efforts have been separately dedicated to big data processing and cross-media mining, with well theoretical underpinnings and great practical success. However, studies jointly considering cross-media big data analytics are relatively sparse. This research gap needs our more attention, since it will benefit lots of real-world applications. Despite its significance and value, it is non-trivial to analyze cross-media big data due to their heterogeneity, large-scale volume, increasing size, unstructured, correlations, and noise. Multi-modal Information Learning, which can be treated as the most significant breakthrough in the past 10 years, has greatly affected the methodology of computer vision and achieved terrific progress in both academy and industry. From then on, deep learning has been adopted in all kinds of computer vision applications and many breakthroughs have achieved in sub-areas, like DeepFace on LFW competition for face verification, GoogleNet for ImageNet Competition for object categorization. It can be expected that more and more computer vision applications will benefit from Multi-modal Information Learning.

The submitted manuscripts were reviewed by experts from both academia and industry. After two rounds of reviewing, the highest quality manuscripts were accepted for this special issue. Totally, we have received 27 manuscripts and 14 papers are accepted in this SI. This special issue will publish by Journal of Ambient Intelligence and Humanized Computing as special issues.

Zheng Xu

xuzheng@shu.edu.cn

1 Shanghai University, 99 Shangda Road, Shanghai 201142, China

2 Changzhou Institute of Mechatronic Technology, Changzhou 213164, Jiangsu, China
The improved neural network is used by Long (2019) to extract the trajectory characteristics of the athletes in the football player's game video, and the network is trained on a large number of data objects containing similarity objects, which improves the ability of the algorithm to distinguish the athlete's trajectory. Li et al. (2019) proposes an intrinsic image decomposition method based on depth learning and probability graph model, in order to extract image information more accurately. Zhao et al. (2019) construct four logistics forums metrics on the degree of busyness and emotional states of the logistics staffs based through hypertext analysis techniques. Liu et al. (2019) employ the VRP with different maturities and the ADL-MIDAS regression model to forecast the expected stock return in Standard \& Poor 500 market. To improve the performance, a neutral mutation (NM) operator for DE algorithm is proposed by Ma et al. (2019). The proposed operator is inspired by neutral theory of molecular evolution, which claims that most mutations are neutral at the level of molecular. Wang et al. (2019a) adopts average cycle analysis method to model analysis and performance index analysis, obtaining expressions of system throughput rate, average delay and communication energy consumption, etc. Yu et al. (2019) proposes a research method for survivability of wireless sensor networks based on tenacity.

The new definitions and methods of attribute circle classification are proposed by Qi et al. (2019). The improvement is using inner attribute polygon and outer attribute polygon to construct the object attribute area. $\mathrm{Wu}$ and $\mathrm{Wu}$ (2019) shows that the methods based on machine learning algorithm modeling such as ridge regression and SVM are suitable for student selection decision modeling. Wenbin et al. (2019) takes scientific research team cooperative network as research object, analyzes the influence of scientific research teams in the cooperative network from the aspect of node heterogeneity and node similarity of content and structure, and puts forward the influence evaluation method of scientific research team. The entropy method is used 
by Ren (2019) to calculate pollution comprehensive index reflecting provincial environmental pollution level. Wang et al. (2019b) describes a hierarchical distribution network reconfiguration strategy with microgrid, which can reduce the number of operation of the switch and the network loss. This strategy ensures rapid power supply recovery. An ontology method based on skin tolerance model is proposed by Peng et al. (2019) in this study to address the abovementioned problems. Zhan et al. (2019) presents cloud insider attack detector and locator (CIADL) on multi-tenant network isolation for OpenStack.

Acknowledgements The guest editors would like to thank Prof. Vincenzo Loia who is the editor in chief of Journal of Ambient Intelligence and Humanized Computing. His help and trust is the most important thing for the success of this SI. The guest editors would like to thank the reviewers for their high quality reviews, which provided insightful and constructive feedback to the authors of the papers.

\section{References}

Li X, Hu G, Pan Z (2019) An eigendecomposition method based on deep learning and probabilistic graph model. J Ambient Intell Hum Comput. https://doi.org/10.1007/s12652-019-01555-0

Liu R, Yang J, Ruan C (2019) Expected stock return and mixed frequency variance risk premium data. J Ambient Intell Hum Comput. https://doi.org/10.1007/s12652-019-01528-3

Long T (2019) Research on application of athlete gesture tracking algorithms based on deep learning. J Ambient Intell Hum Comput. https://doi.org/10.1007/s12652-019-01575-w

Ma C, Liu Y, Wang C et al (2019) A neutral mutated operator applied for DE algorithms. J Ambient Intell Hum Comput. https://doi. org/10.1007/s12652-019-01498-6

Peng Z, Huang M, Zhong Y et al (2019) Construction of ontology for auto-interpretable tolerance semantics in skin model. J Ambient Intell Hum Comput. https://doi.org/10.1007/s12652-019-01497-7
Qi X, Cui T, Shao L et al (2019) Research on intelligent classification of multi-attribute safety information and determination of operating environment. J Ambient Intell Hum Comput. https://doi. org/10.1007/s12652-019-01474-0

Ren Y (2019) Research on the green total factor productivity and its influencing factors based on system GMM model. J Ambient Intell Hum Comput. https://doi.org/10.1007/s12652-019-01472-2

Wang X, Cheng M, Liu Y (2019a) Analysis of MAC protocol of wireless sensor network based on random multi-address access and three-probability joint-control. J Ambient Intell Hum Comput. https://doi.org/10.1007/s12652-019-01539-0

Wang X, Ji Y, Wang J et al (2019b) Research on distribution network reconfiguration based on microgrid. J Ambient Intell Hum Comput. https://doi.org/10.1007/s12652-019-01542-5

Wenbin Z, Tongrang F, Zhixian Y et al (2019) An evaluation method of scientific research team influence based on heterogeneity and node similarity of content and structure. J Ambient Intell Hum Comput. https://doi.org/10.1007/s12652-019-01547-0

Wu X, Wu J (2019) Criteria evaluation and selection in non-native language MBA students admission based on machine learning methods. J Ambient Intell Hum Comput. https://doi.org/10.1007/ s12652-019-01490-0

Yu J, Yu Z, Ding M et al (2019) Research on the tenacity survivability of wireless sensor networks. J Ambient Intell Hum Comput. https ://doi.org/10.1007/s12652-019-01491-z

Zhan J, Fan X, Han J et al (2019) CIADL: cloud insider attack detector and locator on multi-tenant network isolation: an OpenStack case study. J Ambient Intell Hum Comput. https://doi.org/10.1007/ s12652-019-01471-3

Zhao J, Sun N, Cheng W (2019) Logistics forum based prediction on stock index using intelligent data analysis and processing of online web posts. J Ambient Intell Hum Comput. https://doi.org/10.1007/ s12652-019-01520-x

Publisher's Note Springer Nature remains neutral with regard to jurisdictional claims in published maps and institutional affiliations. 